\title{
molecules
}

ISSN 1420-3049

www.mdpi.com/journal/molecules

Article

\section{Molecular Cloning and Characterization of a Xanthone Prenyltransferase from Hypericum calycinum Cell Cultures}

\author{
Tobias Fiesel 1,2,†, Mariam Gaid ${ }^{1,2, \dagger}$, Andreas Müller ${ }^{1}$, Joana Bartels ${ }^{2,3}$, Islam El-Awaad ${ }^{1}$, \\ Till Beuerle ${ }^{1}$, Ludger Ernst ${ }^{4}$, Sönke Behrends ${ }^{2,3}$ and Ludger Beerhues ${ }^{1,2, *}$
}

1 Institute of Pharmaceutical Biology, Technische Universität Braunschweig, Mendelssohnstraße 1, Braunschweig 38106, Germany; E-Mails: t.fiesel@tu-bs.de (T.F.); m.gaid@tu-bs.de (M.G.); der.andreas.mueller@gmx.net (A.M.); i.el-awaad@tu-bs.de (I.E.-A.); t.beuerle@tu-bs.de (T.B.)

2 Center of Pharmaceutical Engineering (PVZ), Technische Universität Braunschweig, Franz-Liszt-Straße 35 A, Braunschweig 38106, Germany; E-Mails: jo.bartels@tu-bs.de (J.B.); s.behrends@tu-bs.de (S.B.)

3 Institute of Pharmacology, Toxicology and Clinical Pharmacy, Technische Universität Braunschweig, Mendelssohnstraße 1, Braunschweig 38106, Germany

4 Central NMR Laboratory, Technische Universität Braunschweig, Hagenring 30, Braunschweig 38106, Germany; E-Mail: 1.ernst@tu-bs.de

$\dagger$ These authors contributed equally to this work.

* Author to whom correspondence should be addressed; E-Mail: 1.beerhues@tu-bs.de; Tel.: +49-531-391-5689; Fax: +49-531-391-8104.

Academic Editor: Thomas J. Schmidt

Received: 30 June 2015 / Accepted: 20 August 2015 / Published: 27 August 2015

Abstract: In plants, prenylation of metabolites is widely distributed to generate compounds
with efficient defense potential and distinct pharmacological activities profitable to human
health. Prenylated compounds are formed by members of the prenyltransferase (PT)
superfamily, which catalyze the addition of prenyl moieties to a variety of acceptor molecules.
Cell cultures of Hypericum calycinum respond to elicitor treatment with the accumulation of
the prenylated xanthone hyperxanthone E. A cDNA encoding a membrane-bound PT (HcPT)
was isolated from a subtracted cDNA library and transcript preparations of $H$. calycinum.
An increase in the HcPT transcript level preceded hyperxanthone E accumulation in cell
cultures of $H$. calycinum treated with elicitor. The HcPT cDNA was functionally characterized
by expression in baculovirus-infected insect cells. The recombinant enzyme catalyzed
biosynthesis of 1,3,6,7-tetrahydroxy-8-prenylxanthone through regiospecific C-8 prenylation of 
1,3,6,7-tetrahydroxyxanthone, indicating its involvement in hyperxanthone $\mathrm{E}$ formation. The enzymatic product shared significant structural features with the previously reported cholinesterase inhibitor $\gamma$-mangostin. Thus, our findings may offer a chance for semisynthesis of new active agents to be involved in the treatment of Alzheimer's disease.

Keywords: aromatic prenyltransferase; xanthone; Hypericum; membrane-bound enzyme; Alzheimer's disease

\section{Introduction}

Xanthones are a class of specialized (secondary) plant metabolites which serve as defense compounds against pathogenic microorganisms and herbivores. They are either constitutively formed or accumulated in response to infection and provide the producing plant with a chemical barrier [1,2]. Many compounds exhibit appealing pharmacological activities, thereby attracting wide interest [3,4]. The xanthone scaffold commonly undergoes various biosynthetic modifications, such as glycosylation and prenylation. While glycosylated xanthones are widely distributed in the plant family Gentianaceae, prenylated xanthones are typical of Clusiaceae and Hypericaceae [5]. The latter families not only contain monoprenylated compounds, but also form a number of polyprenylated polycyclic metabolites possessing bridged skeletons. A number of these chemically complex molecules exhibit intriguing biological activities [6,7]. As observed for many phenolic constituents, the addition of prenyl residues significantly affects their pharmacological properties [8].

Prenylation of aromatic scaffolds is catalyzed by aromatic prenyltransferase (PT) enzymes, which link the shikimate and/or polyketide pathways in plants with the isoprenoid routes [8]. The enzymes that have been studied at the gene level are integral membrane proteins possessing a number of transmembrane domains [9]. Their detailed understanding may pave the way for their involvement in engineering strategies.

To gain insight into the biosynthesis of prenylated xanthones, we use elicitor-treated Hypericum calycinum cell cultures, which accumulate a monoprenylated xanthone phytoalexin, hyperxanthone E (Figure 1) [10]. H. calycinum is closely related to the medicinal plant Hypericum perforatum (St. John's wort; Hypericaceae), extracts of which are widely used for the treatment of mild to moderate depression [11]. The xanthone scaffold is of mixed biosynthetic origin, the two aromatic rings originating from the shikimate and the polyketide pathways (Figure 1). Benzophenone synthase condenses benzoyl-CoA, derived from L-phenylalanine via cinnamoyl-CoA [10], with three molecules of malonyl-CoA [12]. The resulting 2,4,6-trihydroxybenzophenone undergoes cytochrome P450-catalyzed 3'-hydroxylation and heterocyclic ring closure to yield 1,3,7-trihydroxyxanthone [13,14]. Downstream reactions are hydroxylations and prenylations [15,16]. In hyperxanthone E formation, 6-hydroxylation [17] and 8-prenylation are followed by pyran ring formation.

Here we report molecular cloning and functional analysis of a PT cDNA from elicitor-treated $H$. calycinum cell cultures and demonstrate the involvement of the encoded membrane-bound enzyme in the penultimate step of hyperxanthone E biosynthesis. 


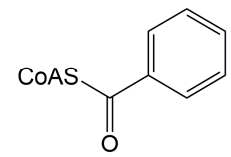

benzoyl-CoA

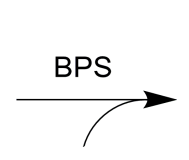

3 malonyl-CoA

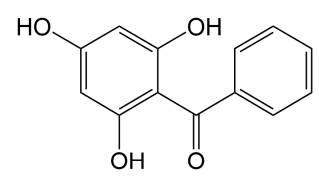

phlorbenzophenone
TXS,

$\mathrm{X} 6 \mathrm{H}$

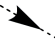
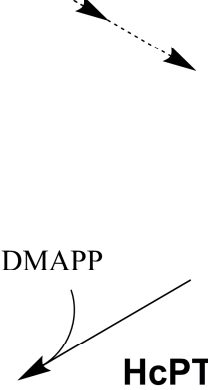

HcPT

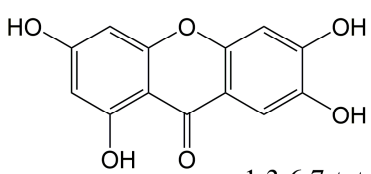

$1,3,6,7$-tetrahydroxyxanthone<smiles>CC1(C)CCc2c(c(O)cc3oc4cc(O)cc(O)c4c(=O)c23)O1</smiles>

hyperxanthone $\mathrm{E}$

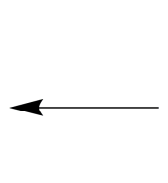

1,3,6,7-tetrahydroxy-8prenylxanthone

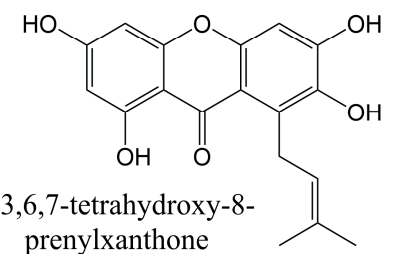

Figure1. Hyperxanthone E biosynthesis in elicitor-treated $H$. calycinum cell cultures. BPS: benzophenone synthase, TXS: trihydroxyxanthone synthase, X6H: xanthone 6-hydroxylase, DMAPP: dimethylallyl diphosphate, HcPT: H. calycinum prenyltransferase.

\section{Results and Discussion}

\subsection{Isolation and Structural Analysis of a cDNA Encoding an Aromatic Prenyltransferase}

A previously constructed subtracted cDNA library [10] was analyzed for putative aromatic prenyltransferase sequences via the Basic Local Alignment Search Tool (tblastx) of the National Center for Biotechnology Information (NCBI) server [18]. Eleven expressed sequence tags (ESTs) were identified and aligned against aromatic prenyltransferase sequences related to secondary metabolism [19-23]. Six of the identified ESTs shared homology with a single sequence contig. This 464-bp middle fragment encoded a peptide with a characteristic motif of aromatic prenyltransferases (Figure 2). A pool of RNA was isolated from elicitor-treated $H$. calycinum cell cultures, reverse-transcribed, and used as a template for re-amplifying the core fragment with the primer pair $1+2$ (Table 1, Supplementary Figure S1). Gene-specific forward and reverse primers $(3+4)$ then served for $5^{\prime}$ and $3^{\prime}$ rapid amplification of cDNA ends (RACE), which led to cloning of a 1535-bp full-length cDNA. The 1191-bp coding sequence (CDS) was flanked by a 65-bp 5' untranslated region (UTR) and a 251-bp 3' UTR plus 28-bp poly(A) tail. The CDS encoded an aromatic prenyltransferase, which was named HcPT and consisted of 396 amino acids with a predicted molecular mass of $43.5 \mathrm{kDa}$ and a $\mathrm{pI}$ of 9.7 [24]. HcPT shared highest similarity (38\%) with predicted homogentisate solanesyl transferase from Populus euphratica (accession number: XP_011047106). The characteristic motifs among aromatic prenyltransferases (motif 1, NQ(I/L)xDxxxD; motif $2, \mathrm{KD}(\mathrm{I} / \mathrm{L}) \mathrm{xDxxGD}$ ) were also conserved in HcPT. The amino acid sequence of HcPT contained six putative transmembrane domains, as predicted by the online tool "SOSUI" (Figure 2) [25]. Furthermore, a putative chloroplast transit peptide of 54 amino acids at the $N$-terminus was predicted by the online tools TargetP (score value: 0.837) and ChloroP (score value: 0.563) [26-28]. A high probability for targeting HcPT to plastids in plant cells was confirmed by the iPSORT online tool [29]. The same held 
true for the aromatic prenyltransferases isolated from Glycine max [21], Humulus lupulus [19,30], Petroselinum crispum [9], and Citrus limon [31].

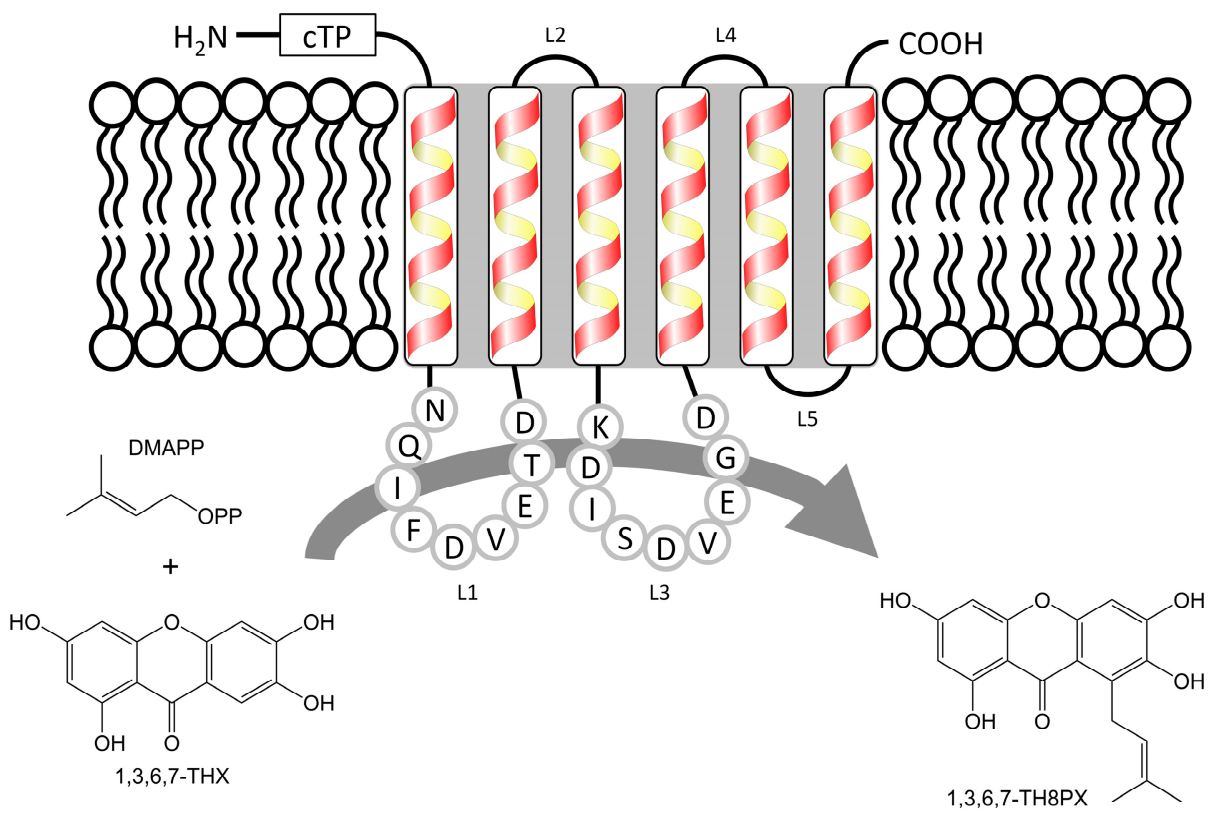

Figure2. HcPT reaction and predicted topology of the transmembrane domains. The two conserved aspartate-rich motifs, which are characteristic for aromatic prenyltransferases and presumably important for the prenylation reaction [20], are located in the non-membrane loop regions L1 and L3. cTP: putative chloroplast transit peptide, L: loop, DMAPP: dimethylallyl diphosphate, 1,3,6,7-THX: 1,3,6,7-tetrahydroxyxanthone, 1,3,6,7-TH8PX: 1,3,6,7-tetrahydroxy-8-prenylxanthone.

Table 1. Primer sequences.

\begin{tabular}{|c|c|}
\hline No. & Sequence * \\
\hline \multicolumn{2}{|c|}{ HcPT-specific cloning primers } \\
\hline 1 & 5'-TAGTACAAGTGATAAGAAATTCGG-3' \\
\hline 2 & 5'-TGTACTTAATGGTAAATCGGGTTTG-3' \\
\hline 3 & 5'-ACTGTATTGACAAGAAGGTTGATGG-3' \\
\hline & 5'-AACGGCATGTATCTTGATGAACATAGG-3' \\
\hline \multicolumn{2}{|r|}{ HcCNL-specific primers for transcript level inspection } \\
\hline 5 & 5'-TCAGCAGCGTGGAGGTCGAGTCG-3' \\
\hline 6 & 5'-TTAAAGACGAGACATGGCAAG-3' \\
\hline \multicolumn{2}{|r|}{ Hc18S-rRNA-specific primers for transcript level normalization } \\
\hline 7 & 5'-TGATGGTATCTACTACTCGG-3' \\
\hline & 5'-AATATACGCTATTGGAGCTGG-3' \\
\hline \multicolumn{2}{|r|}{ HcPT-specific overexpression primers } \\
\hline 9 & 5'-AATGCTAGCATGGAGGTTTCTCGATTGCCATCG-3' \\
\hline 10 & 5'-AATAAGCTTCTAGATGAAGGGGAGCAAGATAAATTGC-3' \\
\hline 11 & 5'-ATTGAATTCATGGAGGTTTCTCGATTGC-3' \\
\hline 12 & 5'-ATTTTAATTAACTAGATGAAGGGGAGCAAGATAAATTGC-3' \\
\hline & 5'-ATTGAATTCATGGAGGTTTCTCGATTGCCATCG-3' \\
\hline
\end{tabular}

* Non-natural endonuclease restriction sites are indicated by underlining. 


\subsection{Elicitor-Induced Changes at the Metabolite and Transcript Levels in H. calycinum Cell Cultures}

Yeast extract-treated $H$. calycinum cell cultures were previously shown to form hyperxanthone E, which started to accumulate $12 \mathrm{~h}$ after the onset of elicitation and reached the peak level equivalent to $4 \mathrm{mg} \cdot \mathrm{g}^{-1}$ dry weight after $20 \mathrm{~h}$ [10]. Biosynthesis of hyperxanthone E was preceded by a transient increase in the HcPT transcript level (Figure 3A). A similar expression profile was observed for HcCNL, whose gene product directs the carbon flow to benzenoid/xanthonoid metabolism (Figure 3B). Changes in the transcript levels were studied by semi-quantitative reverse transcription (RT)-PCR, the conditions being optimized as described previously [10]. The sizes of the PCR products upon use of HcPT and HcCNL gene-specific primers $(1+2$ and $5+6$, respectively; Table 1, Supplementary Figure S1) were 464 and $389 \mathrm{bp}$, respectively. Following elicitation, both transcripts were detectable after four hours and peaked at eight hours. To ensure equal template amounts, H. calycinum 18S rRNA (amplified with the primers $7+8$ ) served as control. The absence of detection of HcPT and HcCNL transcripts before elicitation $(0 \mathrm{~h})$ as well as their increase after the onset of elicitation provide evidence for the involvement of these enzymes in hyperxanthone E biosynthesis.
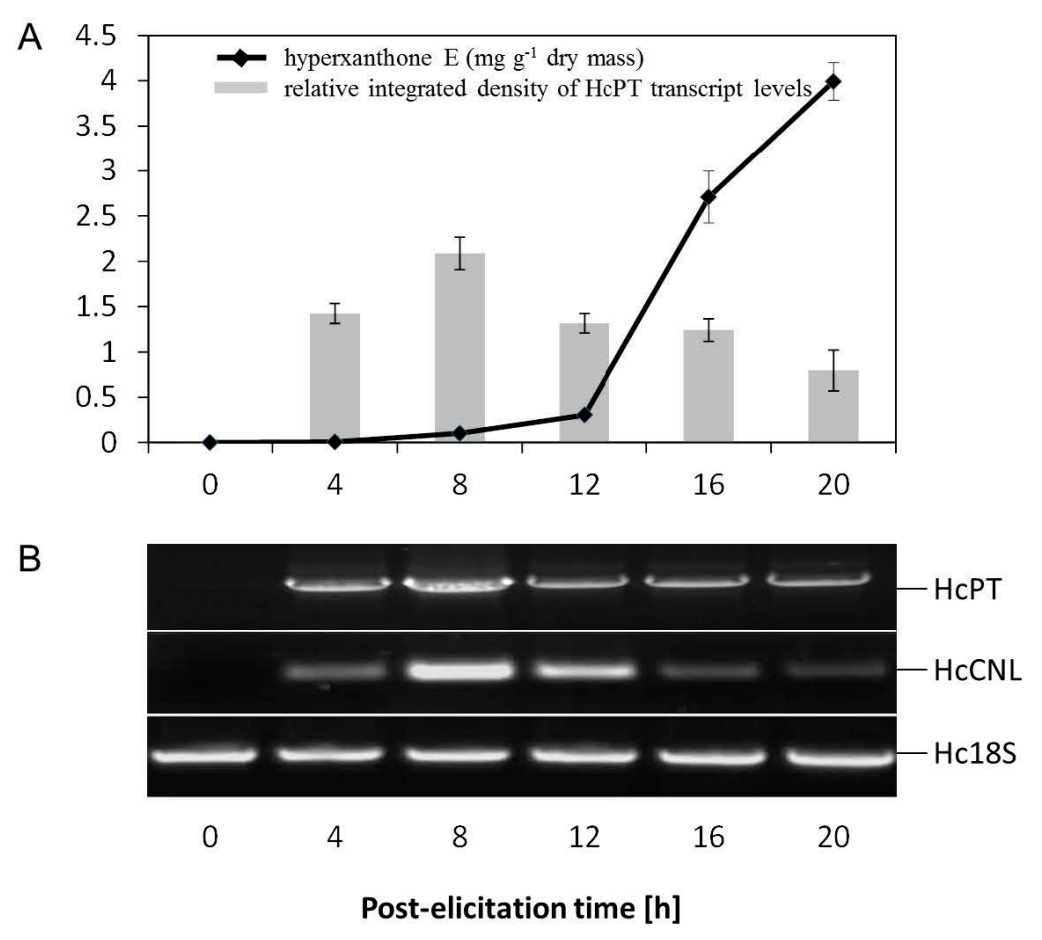

Figure 3. Correlation between gene expression and product formation. (A) Changes in hyperxanthone E content and HcPT transcript level in $H$. calycinum cell cultures after the addition of elicitor. Values are means of three biological repeats \pm SD; (B) Semi-quantitative RT-PCR analysis of HcPT and HcCNL gene expression. Hc18S rRNA transcript levels served for normalization. HcCNL: H. calycinum cinnamate:CoA ligase, Hc18S: H. calycinum 18S rRNA-derived control.

\subsection{Functional Expression of $\mathrm{HcPT}$}

HcPT was predicted to contain multiple transmembrane $\alpha$-helices, suggesting a membranous and hydrophobic protein character. Establishment of this type of protein in prokaryotes is a challenge, 
contrary to the $\beta$-barrel proteins [32], with rare exceptions being reported [33,34]. The presence of $\alpha$-helical membrane-spanning domains requires a special, i.e., eukaryotic, expression system to yield correctly folded protein and circumvent cell death. However, handling of such a hydrophobic protein is a challenge, even in a eukaryotic expression system. Microsomes from yeast (Saccharomyces cerevisiae) cells expressing the HcPT coding sequence failed to prenylate the target substrate. Similar observations were made with aromatic prenyltransferases from Petroselinum crispum and glycinol-4-dimethylallyltransferase from Glycine $\max$ [9,21]. An alternative strategy is to produce HcPT in a baculovirus insect cell (Spodoptera frugiperda, $S f 9$ ) expression system. $p$-Hydroxybenzoate:geranyltransferase - a membranebound enzyme involved in shikonin biosynthesis in Lithospermum erythrorhizon — exhibited $\sim 1000$-fold increased activity upon production in the baculovirus insect cell system compared to expression in yeast [22].

The microsomal fraction from insect cells expressing the HcPT CDS was used for carrying out enzyme assays. Prenylation activity was tested using an array of xanthones, flavonoids, phenolic acids, and benzophenones in the presence of $0.2 \mathrm{mM}$ dimethylallyl diphosphate (DMAPP). Among the examined substrates, 1,3,6,7-tetrahydroxyxanthone was preferred as prenyl acceptor (Supplementary Figure S2B). Product analysis demonstrated that the acceptor was prenylated once, which was detected by LC-MS in comparison with a sample of the authentic reference compound 1,3,6,7-tetrahydroxy-8-prenylxanthone (Supplementary Figure S3). The molecular ion peak $[\mathrm{M}+\mathrm{H}]^{+}$at $m / z 329$ and the MS/MS fragmentation pattern agreed with previous reports [16,35]. In HPLC-DAD, the enzymatic product co-eluted with the reference compound and the UV spectra matched, exhibiting the characteristic absorption bands of a tetraoxygenated xanthone skeleton at $\lambda_{\max } 254,312$, and $360 \mathrm{~nm}$ (Figure 4). Based on the $\mathrm{R}_{\mathrm{t}}$ value, pyran ring formation was excluded (Figure 4C). Assays containing heat-denatured microsomes or the microsomal fraction from cells that harbored the empty vector served as control and lacked enzymatic product formation (Supplementary Figure S2B). For NMR analysis, the enzymatic product was purified from a large-scale incubation by semi-preparative HPLC on a $\mathrm{C}_{18}$ column (Experimental Section 3.10). The ${ }^{1} \mathrm{H}-\mathrm{NMR}$ data (Table 2) agreed with the previously published spectrum of 1,3,6,7-tetrahydroxy-8prenylxanthone [35]. Alternative prenylation at the $\mathrm{C}-5$ position was ruled out [36].

Table 2. ${ }^{1} \mathrm{H}-\mathrm{NMR}$ data of HcPT-formed 1,3,6,7-tetrahydroxy-8-prenylxanthone $(600 \mathrm{MHz}$, acetone- $\left.d_{6} / \mathrm{TMS}\right)$.

\begin{tabular}{ccccc}
\hline Proton Position & $\boldsymbol{\delta H} *$ & $\boldsymbol{J}(\mathbf{H z})$ & \\
\hline $1-\mathrm{OH}$ & $14.03[1 \mathrm{H}]$ & $\mathrm{s}$ & $\mathrm{s}$ \\
$5-\mathrm{H}$ & $6.54[1 \mathrm{H}]$ & $\mathrm{s}$ & \\
$4-\mathrm{H}$ & $6.30[1 \mathrm{H}]$ & $\mathrm{d}, 2$ & \\
$2-\mathrm{H}$ & $6.15[1 \mathrm{H}]$ & $\mathrm{d}, 2$ & $\mathrm{~d}, 6$ & \\
$=\mathrm{CH}-$ & $5.47[1 \mathrm{H}]$ & $\mathrm{t}, 6$ & \\
$8-\mathrm{CH} 2$ & $4.04[2 \mathrm{H}]$ & $\mathrm{d}, 6$ & \\
$=\mathrm{C}(\mathrm{CH} 3)$ & $1.80[3 \mathrm{H}]$ & $\mathrm{s}$ & \\
$=\mathrm{C}(\mathrm{CH} 3)$ & $1.63[3 \mathrm{H}]$ & $\mathrm{s}$ &
\end{tabular}

* The signals of the aromatic protons were doubled $(\Delta \delta=0.002-0.004 \mathrm{ppm})$, presumably because of the partial replacement of the hydroxyl group protons by deuterium due to the simultaneous presence of $\mathrm{H}_{2} \mathrm{O}$ and $\mathrm{D}_{2} \mathrm{O}$ in the solvent (isotope effects upon chemical shifts). 


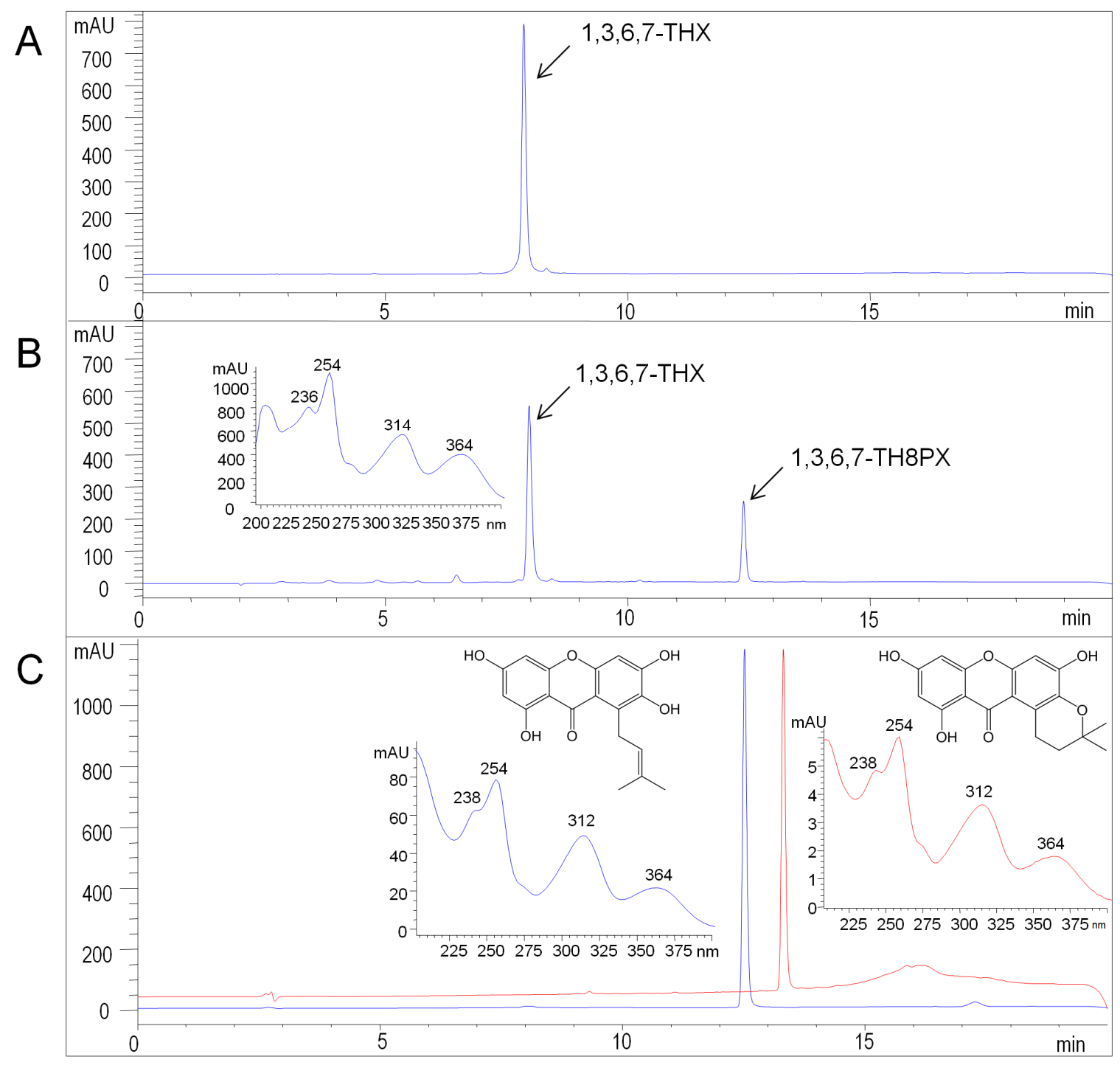

Figure 4. HPLC-DAD analysis of HcPT assays. (A) Incubation with denatured protein; (B) Standard assay incubated for $30 \mathrm{~min}$. Insert: UV spectrum of the enzymatic product 1,3,6,7-TH8PX; (C) Preparative enzymatic synthesis of 1,3,6,7-TH8PX (blue) and chromatography of authentic hyperxanthone E (red). Inserts: UV spectra. 1,3,6,7-THX: 1,3,6,7-tetrahydroxyxanthone, 1,3,6,7-TH8PX: 1,3,6,7-tetrahydroxy-8-prenylxanthone. Detection wavelength: $254 \mathrm{~nm}$.

High $\mathrm{C}-8$ prenylation activity was observed in $50 \mathrm{mM}$ potassium phosphate buffer at $\mathrm{pH} 7.5$ (Supplementary Figure S4A). No enzyme activity was detected in assays that lacked $\mathrm{MgCl}_{2}$, indicating the strict dependence of HcPT on the divalent cation $\mathrm{Mg}^{2+}$. Lower activities were observed with other divalent metal ions (Supplementary Figure S4B). The optimum incubation temperature was at $40{ }^{\circ} \mathrm{C}$ (Supplementary Figure S4C). The $K_{\mathrm{m}}$ values for 1,3,6,7-tetrahydroxyxanthone and DMAPP were $211 \pm 16$ and $87 \pm 5 \mu \mathrm{M}$, respectively, as inferred from a Lineweaver-Burk plot. No appreciable loss in $\mathrm{HcPT}$ activity was observed upon storage at $-80{ }^{\circ} \mathrm{C}$ for two months.

An array of prenylated xanthones, either naturally or synthetically generated, possess interesting pharmacological and biological activities, suggesting that a prenyl residue may significantly contribute [37-39]. An alkyl group at C-2 of 1,3-dihydroxy-2-methylxanthone was important for the 
selectivity against breast adenocarcinoma MCF-7 (Michigan Cancer Foundation-7) cells [40]. Furthermore, the anticholinesterase activity of 8-deoxygartanin was 17 times lower than that of $\gamma$-mangostin, whose IC50 value was $0.45 \mu \mathrm{g} \cdot \mathrm{mL}^{-1}$, compared to $0.27 \mu \mathrm{g} \cdot \mathrm{mL}^{-1}$ for galantamine [41]. The decrease in inhibition was attributed to the absence of the 8-prenyl moiety, as suggested by molecular docking. The enzymatic product of HcPT is a promising precursor for the partial synthesis of compounds that may offer an alternative for management of Alzheimer's disease. Previous attempts to regiospecifically introduce prenyl moieties on the xanthone skeleton resulted in low yields. C-2 and C-4 prenylations of 1,3,5-trihydroxyxanthone were achieved at yields of $3 \%$ and $8 \%$, respectively [42]. Furthermore, a modified method yielded $11 \%$ and $13 \%$, respectively [43]. To date, there is no report about successful synthesis of a $\mathrm{C}-8$ prenylxanthone. HcPT regiospecifically prenylated 1,3,6,7-tetrahydroxyxanthone at C-8 with 70\% substrate conversion within two hours (Supplementary Figure S4D).

\section{Experimental Section}

\subsection{Reagents}

All reagents used were obtained from Roth (Karlsruhe, Germany), Sigma Aldrich (Steinheim, Germany), Acros Organics (Geel, Belgium), and Fisher Chemical (Nidderau, Germany), unless otherwise stated.

\subsection{Synthesis of Trisammonium Dimethylallyl Diphosphate and 1,3,6,7-Tetrahydroxyxanthone}

Trisammonium dimethylallyl diphosphate was synthesized as described previously [44], starting with commercially available dimethylallyl chloride. The substrate 1,3,6,7-tetrahydroxyxanthone was prepared semi-synthetically in a two-step synthesis. In a first step, 1,3,7-trihydroxyxanthone was synthesized according to Genoux-Bastide et al. [45] and purified over a silica gel column (silica gel 60, 0.040-0.063 mm (Merck, Darmstadt, Germany); column dimensions: $10 \times 2.5 \mathrm{~cm}, 20 \mathrm{~g}$ silica gel) using gradient elution (20\%-40\% ethyl acetate in petroleum ether). The intermediate 1,3,7-trihydroxyxanthone was hydroxylated using a genetically modified yeast ( $S$. cerevisiae) strain expressing a xanthone 6-hydroxylase gene. The resulting 1,3,6,7-tetrahydroxyxanthone was extracted from the growth medium and purified using semi-preparative thin layer chromatography (silica gel $\mathrm{C}_{18}-100 \mathrm{UV}_{254}$; methanol/water: 60:40).

\subsection{Plant Material and Elicitation}

Cell suspension cultures of H. calycinum L. (Hypericaceae) were generated as described previously [46]. For propagation, aliquots of $3 \mathrm{~g}$ of suction-dried cells were transferred every two weeks into $50 \mathrm{~mL}$ of liquid Linsmaier and Skoog (LS) medium [47]. Cultures were shaken in Erlenmeyer flasks at $125 \mathrm{rpm}$ and $25{ }^{\circ} \mathrm{C}$ in the dark. Four-day-old cell cultures were treated with yeast extract as an elicitor $\left(3 \mathrm{~g} \cdot \mathrm{L}^{-1}\right)$ and harvested $8 \mathrm{~h}$ post-elicitation.

\subsection{Isolation of an HcPT $c D N A$}

Eight hours after yeast extract treatment, $H$. calycinum cell cultures were harvested by vacuum filtration and ground to fine powder under liquid nitrogen. Total RNA was extracted from $100 \mathrm{mg}$ of homogenized plant material using the RNeasy Plant Mini Kit (Qiagen, Hilden, Germany). Oligo(dT)-primed 
reverse transcription was carried out at $42{ }^{\circ} \mathrm{C}$ with $1 \mu \mathrm{g}$ total RNA and SuperScript II Reverse Transcriptase (Invitrogen, Karlsruhe, Germany). The synthesized cDNA pool served as template for amplifying the HcPT core fragment using the gene-specific primers $1+2$ (Table 1, Supplementary Figure S1) and peqGOLD Taq-DNA-Polymerase (peqlab, Erlangen, Germany). Design of the primer pair was based on sequence information from a subtracted cDNA library [10]. After denaturation at $94{ }^{\circ} \mathrm{C}(2 \mathrm{~min}), 30$ cycles were carried out at $94{ }^{\circ} \mathrm{C}(30 \mathrm{~s}), 55^{\circ} \mathrm{C}(45 \mathrm{~s})$, and $72{ }^{\circ} \mathrm{C}(2 \mathrm{~min})$, followed by final extension at $72{ }^{\circ} \mathrm{C}$ for $10 \mathrm{~min}$. The core fragment was then extended toward the $5^{\prime}$ and $3^{\prime}$ ends by rapid amplification of cDNA ends (RACE), using the protocol of the SMARTer RACE cDNA Amplification Kit (Clontech, Heidelberg, Germany) and the gene-specific primers $3+4$ (Table 1, Supplementary Figure S1). The resulting amplicons were cloned into the pGEM-T Easy vector (Promega, Mannheim, Germany) and sequence information was obtained by DNA sequencing at GATC-Biotech (Konstanz, Germany).

\subsection{Semi-Quantitative Reverse Transcription PCR}

At defined times ( $0-20 \mathrm{~h}$ ) after yeast treatment, total RNA was isolated from cultured $H$. calycinum cells $(0.5 \mathrm{mg})$. Reverse transcription and PCR were carried out as described above under section 3.4 except that the annealing time and amplification cycles were reduced to $30 \mathrm{~s}$ and 28 cycles, respectively. Primer pair $1+2$ (Table 1, Supplementary Figure S1) was used to analyze HcPT transcripts. To examine the HcCNL transcript level, primers $5+6$ were used. The 18S ribosomal DNA (Hypericum rDNA; AF206934) transcripts served to normalize RT-PCR results using primer pair $7+8$. Annealing temperatures for HcPT, HcCNL and $18 \mathrm{~S}$ were 55,60 and $51^{\circ} \mathrm{C}$, respectively. The PCR products were finally analyzed as described under 3.4 using the free open source image processing program ImageJ (version 1.36, http://rsb.info.nih.gov/ij/) [10].

\subsection{Heterologous Expression of HcPT}

Within the first expression trials, prokaryotic cells from the LEMO21 (DE3) system (New England Biolabs, Frankfurt, Germany) were used according to the manufacturer's protocol using the pRSET-B expression vector and the overexpression primers $9+10$ (Table 1, Supplementary Figure S1). HcPT expression in the yeast strain INVSc1 (S. cerevisiae, Invitrogen, Karlsruhe, Germany) was carried out as described elsewhere [48]. The expression plasmid pESC-URA and a primer pair $11+12$ with an EcoRI and a $\mathrm{PacI}$ restriction site were used for cloning and expression analysis. For overexpression in insect cells (S. frugiperda, Sf9), the HcPT CDS was amplified with Phusion Hot Start II High-Fidelity DNA Polymerase (Thermo Scientific, Dreieich, Germany) using the primers $13+14$ with an EcoRI restriction site and a HindIII restriction site, respectively. PCR included initial denaturation at $98{ }^{\circ} \mathrm{C}(30 \mathrm{~s})$, 35 cycles with $98^{\circ} \mathrm{C}(10 \mathrm{~s}), 66.1^{\circ} \mathrm{C}(30 \mathrm{~s})$, and $72{ }^{\circ} \mathrm{C}(60 \mathrm{~s})$, followed by final extension at $72{ }^{\circ} \mathrm{C}(10 \mathrm{~min})$. Subcloning into the pFastBac1 expression vector (Invitrogen, Karlsruhe, Germany) was made by ligating the EcoRI-HindIII-digested PCR product into EcoRI-HindIII-linearized pFastBac1, generating the expression plasmid pFastBac1-HcPT. The nucleotide sequence of the expression construct was verified by DNA sequencing. HcPT-baculovirus was generated according to the Bac-to-Bac Baculovirus Expression System manual (Invitrogen, Karlsruhe, Germany). For cultivation of Sf9 insect cells, Sf-900 II serum-free medium was supplemented with $1 \%$ penicillin/streptomycin and $10 \%$ fetal calf serum. Insect cell cultures were shaken at $27{ }^{\circ} \mathrm{C}$ at $140 \mathrm{rpm}$ and were diluted to a concentration of 
$2 \times 10^{6}$ cells $\mathrm{mL}^{-1}$ for infection. Cell suspension volumes between 20 and $200 \mathrm{~mL}$ were infected with an HcPT-baculovirus stock.

\subsection{Microsomal Preparation from Insect Cells}

Microsomes were isolated as described previously [22], except for minor modifications. The infected cell suspension was transferred into $50 \mathrm{~mL}$ tubes and was pelleted at $2500 \times \mathrm{g}$ for $5 \mathrm{~min}$ at $4{ }^{\circ} \mathrm{C}$. The pellet was re-suspended in $5 \mathrm{~mL}$ of sonication buffer. The resulting cell suspension was sonicated for $60 \mathrm{~s}$ three times (duty cycle: 15\%, output control: 1.5) using a Sonifier 250 (Branson, Danbury, CT, USA). After centrifugation under the above conditions for removing cell debris and nuclei, the microsomal pellet was obtained by ultracentrifugation of the supernatant at $100,000 \times g$ for $1 \mathrm{~h}$ at $4{ }^{\circ} \mathrm{C}$. The pellet was re-suspended in $2 \mathrm{~mL}$ of ice-cold buffer A by pipetting with syringe and needle to form the enzyme solution for the PT assay. Protein concentrations were determined by the Bradford method [49] with bovine serum albumin as standard.

\subsection{In Vitro Enzyme Assay}

The standard PT incubation contained $0.2 \mathrm{mM}$ 1,3,6,7-tetrahydroxyxanthone, $0.2 \mathrm{mM}$ dimethylallyl diphosphate, $4 \mathrm{mM} \mathrm{MgCl} 2,0.5 \mathrm{mM}$ dithiothreitol, and an aliquot of the microsomal fraction containing around $100 \mu \mathrm{g}$ protein. The final assay volume was adjusted to $250 \mu \mathrm{L}$ with $50 \mathrm{mM}$ potassium phosphate buffer ( $\mathrm{pH} 7.5$ ). After incubation at $40{ }^{\circ} \mathrm{C}$ for $30 \mathrm{~min}$, the reaction was stopped by the addition of $25 \mu \mathrm{L}$ glacial acetic acid $(20 \%$ in methanol). The reaction mixture was extracted twice with ethyl acetate (500 and $250 \mu \mathrm{L}$ ) and the combined organic phase was evaporated to dryness under an air stream at room temperature. The residue was dissolved in $80 \mu \mathrm{L}$ methanol (HPLC grade) and analyzed by HPLC. To determine the $K_{\mathrm{m}}$ value for the substrate 1,3,6,7-tetrahydroxyxanthone, concentrations from 5 to $1000 \mu \mathrm{M}$ were used, while keeping the DMAPP concentration constant at $400 \mu \mathrm{M}$. To measure the $K_{\mathrm{m}}$ value for the prenyl donor DMAPP, the concentration varied from 5-500 $\mu \mathrm{M}$, with the 1,3,6,7-tetrahydroxyxanthone level being constant at $500 \mu \mathrm{M}$.

\subsection{HPLC and LC-ESI-MS Analyses}

The reaction product was analyzed by HPLC (Agilent 1260 Infinity Quaternary LC System; Agilent Technologies, Santa Clara, CA, USA) equipped with a diode array detector (G4212A). A ZORBAX SB-C 18 column $(3.5 \mu \mathrm{m}, 4.6 \times 150 \mathrm{~mm}$; Agilent $)$ was used. The mobile phase consisted of water acidified with $1 \mathrm{mM}$ formic acid and methanol. For standard analysis at a flow rate of $0.5 \mathrm{~mL} \cdot \mathrm{min}^{-1}$, the methanol concentration changed as follows: $50 \%-75 \%$ in $5 \mathrm{~min}, 75 \%-90 \%$ in $10 \mathrm{~min}$, and $95 \%$ in $5 \mathrm{~min}$. To analyze incubations containing 1,7-dihydroxyxanthone as a substrate, methanol was replaced with acetonitrile in the following gradient: $40 \%-70 \%$ in $5 \mathrm{~min}, 70 \%-92 \%$ in $5 \mathrm{~min}$, and $92 \%-95 \%$ in $15 \mathrm{~min}$. The detection wavelength used for monitoring the elution of both 1,3,6,7-tetrahydroxyxanthone and its prenylated product was $254 \mathrm{~nm}$. To analyze the potential conversion of other substrates, the incubation time was elongated to $2 \mathrm{~h}$ and corresponding absorption maxima were chosen as detection wavelengths. 1,3,6,7-Tetrahydroxy-8-prenylxanthone was identified by ESI-MS analysis, as described previously [10]. The molecular ion peak $[\mathrm{M}+\mathrm{H}]^{+}$of the enzymatic product was further analyzed by MS/MS experiments 
in the enhanced product ion mode of the instrument using nitrogen gas for collision-induced dissociation at the high-level setting. The Analyst software (version 1.4.2; Applied Biosystems/MDS Sciex, Waltham, MA, USA) served for data acquisition and processing (Supplementary Figure S3).

\subsection{0. ${ }^{1} H-N M R$ Analysis}

To achieve sufficient amounts of 1,3,6,7-tetrahydroxy-8-prenylxanthone, the volume of the standard enzyme assay was increased 200 -fold and the incubation time at $40{ }^{\circ} \mathrm{C}$ was extended to $20 \mathrm{~h}$, adding after $3 \mathrm{~h}$ another $25 \%$ protein and DMAPP relative to the starting amounts. ${ }^{1} \mathrm{H}-\mathrm{NMR}$ analysis was carried out on a Bruker Avance II 600 spectrometer using acetone- $d_{6}$ (Deutero, Kastellaun, Germany) as solvent and $20{ }^{\circ} \mathrm{C}$ as sample temperature.

\section{Conclusions}

An aromatic prenyltransferase (HcPT) specific for enzymatic alkylation of xanthones has been isolated from elicitor-treated $H$. calycinum cell cultures. The enzyme regiospecifically introduced a prenyl side chain at $\mathrm{C}-8$ of the dibenzo- $\gamma$-pyrone skeleton. Recently, classification and chemical synthesis of simple and prenylated xanthones have been reviewed [50-53] and divergent biological activities have been reported [54-56]. The HcPT product 1,3,6,7-tetrahydroxy-8-prenylxanthone shares structural features with $\gamma$-mangostin, a potent inhibitor of cholinesterase. The importance of the 8-prenyl group was revealed by docking studies [41]. Thus, HcPT offers an interesting tool to provide a precursor for semisynthesis of pharmacologically active compounds with the potential to be used in the treatment of Alzheimer's disease. In addition, further xanthone-specific prenyltransferases need to be studied at the gene level to pave the way for biotechnological production of a wider array of compounds, which may improve the therapy of neurodegenerative disorders.

Sequence data from this article can be found in the GenBank/EMBL data libraries under the accession number KT325851.

\section{Supplementary Materials}

Supplementary materials can be accessed at: http://www.mdpi.com/1420-3049/20/09/15616/s1.

\section{Acknowledgments}

This work was supported by grants from the Deutsche Forschungsgemeinschaft (DFG) and the joint project SynFoBiA of the Center of Pharmaceutical Engineering (PVZ).

\section{Author Contributions}

All authors contributed to the appearance of this article. Conception of experiments was designed by T.F., M.G., A.M., L.B., experimental work was accomplished by T.F., M.G., A.M., I.E.-A., results and analytical data were evaluated by T.F., M.G., A.M., T.B., L.E., L.B., establishment and maintenance of the insect cell expression system were contributed by J.B., S.B., and the manuscript was prepared by T.F., M.G. and L.B. 


\section{Conflicts of Interest}

The authors declare no conflict of interest.

\section{References}

1. Beerhues, L. Biosynthesis of the Active Hypericum perforatum Constituents. In Medicinal and Aromatic Plant Science and Biotechnology; Odabas, M.S., Cirak, C., Eds.; Global Science Books: Isleworth, UK, 2011; Volume 5 (Special Issue 1: Hypericum), pp. 70-77.

2. Tocci, N.; Simonetti, G.; D’Auria, F.D.; Panella, S.; Palamara, A.N.; Valletta, A.; Pasqua, G. Root cultures of Hypericum perforatum subsp. angustifolium elicited with chitosan and production of xanthone-rich extracts with antifungal activity. Appl. Microbiol. Biotechnol. 2011, 91, 977-987.

3. El-Seedi, H.R.; El-Barbary, M.A.; El-Ghorab, D.M.H.; Bohlin, L.; Borg-Karlson, A.K.; Goransson, U.; Verpoorte, R. Recent insights into the biosynthesis and biological activities of natural xanthones. Curr. Med. Chem. 2010, 17, 854-901.

4. Na, Y. Recent cancer drug development with xanthone structures. J. Pharm. Pharmacol. 2009, 61, 707-712.

5. Bennett, G.J.; Lee, H.H. Xanthones from Guttiferae. Phytochemistry 1989, 28, 967-998.

6. Chantarasriwong, O.; Cho, W.C.; Batova, A.; Chavasiri, W.; Moore, C.; Rheingold, A.L.; Theodorakis, E.A. Evaluation of the pharmacophoric motif of the caged Garcinia xanthones. Org. Biomol. Chem. 2009, 7, 4886-4894.

7. Anantachoke, N.; Tuchinda, P.; Kuhakarn, C.; Pohmakotr, M.; Reutrakul, V. Prenylated caged xanthones: Chemistry and biology. Pharm. Biol. 2012, 50, 78-91.

8. Yazaki, K.; Sasaki, K.; Tsurumaru, Y. Prenylation of aromatic compounds, a key diversification of plant secondary metabolites. Phytochemistry 2009, 70, 1739-1745.

9. Karamat, F.; Olry, A.; Munakata, R.; Koeduka, T.; Sugiyama, A.; Paris, C.; Hehn, A.; Bourgaud, F.; Yazaki, K. A coumarin-specific prenyltransferase catalyzes the crucial biosynthetic reaction for furanocoumarin formation in parsley. Plant J. 2014, 77, 627-638.

10. Gaid, M.M.; Sircar, D.; Müller, A.; Beuerle, T.; Liu, B.; Ernst, L.; Hänsch, R.; Beerhues, L. Cinnamate:CoA ligase initiates the biosynthesis of a benzoate-derived xanthone phytoalexin in Hypericum calycinum cell cultures. Plant Physiol. 2012, 160, 1267-1280.

11. Russo, E.; Scicchitano, F.; Whalley, B.J.; Mazzitello, C.; Ciriaco, M.; Esposito, S.; Patanè, M.; Upton, R.; Pugliese, M.; Chimirri, S.; et al. Hypericum perforatum: Pharmacokinetic, mechanism of action, tolerability, and clinical drug-drug interactions. Phytother. Res. 2014, 28, 643-655.

12. Liu, B.; Falkenstein-Paul, H.; Schmidt, W.; Beerhues, L. Benzophenone synthase and chalcone synthase from Hypericum androsaemum cell cultures: cDNA cloning, functional expression, and site-directed mutagenesis of two polyketide synthases. Plant J. 2003, 34, 847-855.

13. Peters, S.; Schmidt, W.; Beerhues, L. Regioselective oxidative phenol couplings of 2,3',4,6-tetrahydroxybenzophenone in cell cultures of Centaurium erythraea RAFN and Hypericum androsaemum L. Planta 1997, 204, 64-69.

14. Schmidt, W.; Beerhues, L. Alternative pathways of xanthone biosynthesis in cell cultures of Hypericum androsaemum L. FEBS Lett. 1997, 420, 143-146. 
15. Beerhues, L.; Berger, U. Xanthones in cell suspension cultures of two Centaurium species. Phytochemistry 1994, 35, 1227-1231.

16. Schmidt, W.; Abd El-Mawla, M.A.; Wolfender, J.L.; Hostettmann, K.; Beerhues, L. Xanthones in cell cultures of Hypericum androsaemum. Planta Med. 2000, 66, 380-381.

17. Schmidt, W.; Peters, S.; Beerhues, L. Xanthone 6-hydroxylase from cell cultures of Centaurium erythraea RAFN and Hypericum androsaemum L. Phytochemistry 2000, 53, 427-431.

18. Basic Local Alignment Search Tool. Available online: http://blast.ncbi.nlm.nih.gov/Blast.cgi (accessed on 3 June 2015).

19. Tsurumaru, Y.; Sasaki, K.; Miyawaki, T.; Uto, Y.; Momma, T.; Umemoto, N.; Momose, M.; Yazaki, K. HlPT-1, a membrane-bound prenyltransferase responsible for the biosynthesis of bitter acids in hops. Biochem. Biophys. Res. Commun. 2012, 417, 393-398.

20. Sasaki, S.; Mito, K.; Ohara, K.; Yamamoto, H.; Yazaki, K. Cloning and characterization of naringenin 8-prenyltransferase, a flavonoid-specific prenyltransferase of Sophora flavescens. Plant Physiol. 2008, 146, 1075-1084.

21. Akashi, T.; Sasaki, K.; Aoki, T.; Ayabe, S.; Yazaki, K. Molecular cloning and characterization of a cDNA for pterocarpan 4-dimethylallyltransferase catalyzing the key prenylation step in the biosynthesis of glyceollin, a soybean phytoalexin. Plant Physiol. 2009, 149, 683-693.

22. Ohara, K.; Mito, K.; Yazaki, K. Homogeneous purification and characterization of LePGT1A membrane-bound aromatic substrate prenyltransferase involved in secondary metabolism of Lithospermum erythrorhizon. FEBS J. 2013, 280, 2572-2580.

23. Ohara, K.; Muroya, A.; Fukushima, N.; Yazaki, K. Functional characterization of LePGT1, a membrane-bound prenyltransferase involved in the geranylation of $p$-hydroxybenzoic acid. Biochem. J. 2009, 421, 231-241.

24. Compute pI/Mw Tool. Available online: http://web.expasy.org/compute_pi (accessed on 3 June 2015).

25. Membrane Topology Prediction. Available online: http://harrier.nagahama-i-bio.ac.jp/sosui/ sosui_submit.html (accessed on 3 June 2015).

26. Subcellular Location Prediction. Available online: http://www.cbs.dtu.dk/services/TargetP (accessed on 3 June 2015).

27. Chloroplast Transit Peptide Prediction. Available online: http://www.cbs.dtu.dk/services/ChloroP (accessed on 3 June 2015).

28. Emanuelsson, O.; Brunak, S.; von Heijne, G.; Nielsen, H. Locating proteins in the cell using TargetP, SignalP and related tools. Nat. Protoc. 2007, 2, 953-971.

29. Localization Prediction for $N$-Terminal Sorting Signals. Available online: http://ipsort.hgc.jp (accessed on 3 June 2015).

30. Tsurumaru, Y.; Sasaki, K.; Miyawaki, T.; Momma, T.; Umemoto, N.; Yazaki, K. An aromatic prenyltransferase-like gene HIPT-1 preferentially expressed in lupulin glands of hop. Plant Biotechnol. 2010, 27, 199-204.

31. Munakata, R.; Inoue, T.; Koeduka, T.; Karamat, F.; Olry, A.; Sugiyama, A.; Takanashi, K.; Dugrand, A.; Froelicher, Y.; Tanaka, R.; et al. Molecular cloning and characterization of a geranyl diphosphate-specific aromatic prenyltransferase from lemon. Plant Physiol. 2014, 166, 80-90.

32. Buchanan, S.K. Beta-barrel proteins from bacterial outer membranes: Structure, function and refolding. Curr. Opin. Struct. Biol. 1999, 9, 455-461. 
33. Rogl, H.; Kosemund, K.; Kuhlbrandt, W.; Collinson, I. Refolding of Escherichia coli produced membrane protein inclusion bodies immobilized by nickel chelating chromatography. FEBS Lett. 1998, 432, 21-26.

34. Kiefer, H. In vitro folding of alpha-helical membrane proteins. Biochim. Biophys. Acta 2003, 1610, $57-62$.

35. Nielsen, H.; Arends, P. Xanthone constituents of Hypericum androsaemum. J. Nat. Prod. 1979, 42, 301-304.

36. Mahabusarakam, W.; Rattanaburi, S.; Phongpaichit, S.; Kanjana-Opas, A. Antibacterial and cytotoxic xanthones from Cratoxylum cochinchinense. Phytochem. Lett. 2008, 1, 211-214.

37. Chin, Y.W.; Kinghorn, A.D. Structural characterization, biological effects, and synthetic studies on xanthones from mangosteen (Garcinia mangostana), a popular botanical dietary supplement. Mini Rev. Org. Chem. 2008, 5, 355-364.

38. Palmeira, A.; Paiva, A.; Sousa, E.; Seca, H.; Almeida, G.M.; Lima, R.T.; Fernandes, M.X.; Pinto, M.; Vasconcelos, M.H. Insights into the in vitro antitumor mechanism of action of a new pyranoxanthone. Chem. Biol. Drug Des. 2010, 76, 43-58.

39. Pinto, M.M.M.; Castanheiro, R. Natural prenylated xanthones: Chemistry and biological activities. In Natural Products: Chemistry, Biochemistry and Pharmacology, 1st ed.; Brahmachari, G., Ed.; Narosa Publishing House Pvt. Ltd: New Delhi, India, 2009; pp. 520-675.

40. Castanheiro, R.A.P.; Pinto, M.M.M.; Silva, A.M.S.; Cravo, S.M.M.; Gales, L.; Damas, A.M.; Nazareth, N.; Nascimentoa, M.S.J.; Graham, E. Dihydroxyxanthone prenylated derivatives: Synthesis, structure elucidation, and growth inhibitory activity on human tumor cell lines with improvement of selectivity for MCF-7. Bioorg. Med. Chem. 2007, 15, 6080-6088.

41. Khaw, K.K.; Choi, S.B.; Tanc, S.C.; Wahab, H.A.; Chane, K.L.; Murugaiyaha, V. Prenylated xanthones from mangosteen as promising cholinesterase inhibitors and their molecular docking studies. Phytomedicine 2014, 21, 1303-1309.

42. Gujral, V.; Gupta, S.R. Synthesis of some 3-methylbut-2-enylated 1,3,5-trihydroxyxanthones. Bull. Chem. Soc. Jpn. 1979, 52, 3679-3681.

43. Helesbeux, J.J.; Duval, O.; Dartiguelongue, C.; Séraphin, D.; Oger, J.M.; Richomme, P. Synthesis of 2-hydroxy-3-methylbut-3-enyl substituted coumarins and xanthones as natural products. Application of the Schenck ene reaction of singlet oxygen with ortho-prenylphenol precursors. Tetrahedron 2004, 60, 2293-2300.

44. Woodside, A.B.; Huang, Z.; Poulter, C.D. Trisammonium geranyl diphosphate. Org. Synth. 1988, 66, 211, doi:10.15227/orgsyn.066.0211.

45. Genoux-Bastide, E.; Lorendeau, D.; Nicolle, E.; Yahiaoui, S.; Magnard, S.; di Pietro, A.; Baubichon-Cortay, H.; Boumendjel, A. Identification of xanthones as selective killers of cancer cells overexpressing the ABC transporter MRP1. Chem. Med. Chem. 2011, 6, 1478-1484.

46. Klingauf, P.; Beuerle, T.; Mellenthin, A.; El-Moghazy, S.A.M.; Boubakir, Z.; Beerhues, L. Biosynthesis of the hyperforin skeleton in Hypericum calycinum cell cultures. Phytochemistry 2005, 66, 139-145.

47. Linsmaier, E.M.; Skoog, F. Organic growth factor requirements of tobacco tissue cultures. Physiol. Plant 1965, 18, 100-127. 
48. Sircar, D.; Gaid, M.M.; Chizzali, C.; Reckwell, D.; Kaufholdt, D.; Beuerle, T.; Broggini, G.A.L.; Flachowsky, H.; Liu, B.; Hänsch, R.; et al. Biphenyl-4-hydroxylases involved in aucuparin biosynthesis in rowan and apple are CYP736A proteins. Plant Physiol. 2015, 168, 428-442.

49. Bradford, M.M. A rapid and sensitive method for the quantitation of microgram quantities of protein utilizing the principle of protein-dye binding. Anal. Biochem. 1976, 72, 248-254.

50. Peres, V.; Nagem, T.J. Trioxygenated naturally occurring xanthones. Phytochemistry 1997, 44, 191-214.

51. Peres, V.; Nagem, T.J.; de Oliveira, F.F. Tetraoxygenated naturally occurring xanthones. Phytochemistry 2000, 55, 683-710.

52. Sousa, M.E.; Pinto, M.M.M. Synthesis of xanthones: An overview. Curr. Med. Chem. 2005, 12, 2447-2479.

53. Zhang, X.; Li, X.; Ye, S.; Zhang, Y.; Tao, L.; Gao, Y.; Gong, D.; Xi, M.; Meng, H.; Zhang, M.; et al. Synthesis, SAR and biological evaluation of natural and non-natural hydroxylated and prenylated xanthones as antitumor agents. Med. Chem. 2012, 8, 1012-1025.

54. Pinto, M.M.; Sousa, M.E.; Nascimento, M.S. Xanthone derivatives: New insights in biological activities. Curr. Med. Chem. 2005, 12, 2517-2538.

55. Fotie, J.; Bohle, D.S. Pharmacological and biological activities of xanthones. Anti-Infect. Agents Med. Chem. 2006, 5, 15-31.

56. Xu, Z.; Huang, L.; Chen, X.H.; Zhu, X.F.; Qian X.J.; Feng, G.K.; Lan, W.J.; Li, H.J. Cytotoxic prenylated xanthones from the pericarp of Garcinia mangostana. Molecules 2014, 19, 1820-1827.

Sample Availability: Samples of the compounds 1,3,6,7-tetrahydroxyxanthone and 1,3,6,7-tetrahydroxy8-prenylxanthone are available from the authors.

(C) 2015 by the authors; licensee MDPI, Basel, Switzerland. This article is an open access article distributed under the terms and conditions of the Creative Commons Attribution license (http://creativecommons.org/licenses/by/4.0/). 